\title{
BMJ Open Game jams for cultural safety training in Colombian medical education: a pilot randomised controlled trial
}

\author{
Juan Pimentel (i) , ${ }^{1,2,3}$ Anne Cockcroft, ${ }^{1,4}$ Neil Andersson ${ }^{1,4}$
}

To cite: Pimentel J, Cockcroft A, Andersson N. Game jams for cultural safety training in Colombian medical education: a pilot randomised controlled trial. BMJ Open 2021;11:e042892. doi:10.1136/ bmjopen-2020-042892

- Prepublication history and additional supplemental material for this paper are available online. To view these files, please visit the journal online (http://dx.doi.org/10.1136/ bmjopen-2020-042892).

Received 17 July 2020 Revised 17 February 2021 Accepted 25 April 2021

\section{Check for updates}

(c) Author(s) (or their employer(s)) 2021. Re-use permitted under CC BY-NC. No commercial re-use. See rights and permissions. Published by BMJ.

${ }^{1}$ CIET-PRAM, Department of Family Medicine, McGill University, Montreal, Québec, Canada

${ }^{2}$ Departamento de Medicina Familiar y Salud Pública, Universidad de La Sabana, Chía, Colombia

${ }^{3}$ Grupo de Estudios en Sistemas Tradicionales de Salud, Universidad del Rosario, Bogotá, Colombia

${ }^{4}$ Centro de Investigación de Enfermedades Tropicales (CIET), Universidad Autónoma de Guerrero, Acapulco, Mexico

Correspondence to

Dr Juan Pimentel;

juan.pimentel@mail.mcgill.ca

\section{ABSTRACT}

Objectives Explore the acceptability and feasibility of a randomised controlled trial (RCT) to assess game jamsparticipatory events to cocreate digital or board games in a time-constrained environment-in cultural safety training of medical students. The pilot tests methods and procedures and explores the validity and reliability of our research instrument.

Design Two-arm parallel-group pilot RCT with a 1:1 allocation ratio.

Setting Faculty of Medicine in Chia, Colombia. Participants 79 final-year medical students completed the baseline questionnaire. 64 completed the assessment immediately after the intervention: 31 in the intervention group (20 female) and 33 in the control group (18 female). 35 completed the final assessment (18 control and 17 intervention) 4 months after the intervention.

Interventions The intervention group joined a 5-hour game jam composed of a 1-hour lecture and a 4hour session to create and to play educational games about cultural safety. The control group had a 1-hour conventional lesson, followed by a 4-hour study session of selected readings on cultural safety.

Primary and secondary outcome measures The instrument, an online self-administered Likert-type questionnaire, assessed a self-reported cultural safety results chain based on a planned behaviour theory. Student recruitment, retention and perception of the activity determined acceptability. The methodological and logistical factors for a full-scale study determined feasibility.

Results After the intervention, students randomised to that arm reported a slightly higher cultural safety score (26.9) than those in the control group (25.9) (difference $-1,95 \% \mathrm{Cl}-3.0$ to 1.0 ). Students described game jam learning in favourable terms and considered cultural safety training relevant. The university authorised the conduct of the full-scale trial.

Conclusion Game jam learning is feasible and acceptable for cultural safety training of Colombian medical students. Researchers and educators may find our results informative in the design of RCTs assessing educational interventions.

Trial registration number ISRCTN14261595 (stage: pilot study results)

\section{INTRODUCTION}

Ignoring the role of culture in health and healthcare is an integral part of the stigma

\section{Strengths and limitations of this study}

This is the first randomised controlled trial exploring game jam learning in medical education and the first quantitative study exploring cultural safety training in Latin America.

Innovative research methods included probabilistic transitive closure.

- We tested the validity and reliability of the instrument for use in the full trial.

- This pilot study was underpowered to detect differences within and between study arms.

and discrimination towards culturally diverse patients. ${ }^{1}$ International institutions have called for more attention to the relationship between culture and health outcomes in medical education. ${ }^{2}$ Contemporary cultural training programmes for health professionals use a range of approaches including cultural competence, sensitivity, awareness, safety, cross-cultural and transcultural medicine, and culturally responsive care. ${ }^{3}$

Cultural competence is 'a set of congruent behaviours, attitudes, and policies that come together in a system, agency, or among professionals and enable that system, agency, or those professionals to work effectively in cross-cultural situations' (p12). ${ }^{4}$ This is the most common cultural training approach. Examples of cultural competence include ethnic matching of physician and patient, and clinician training in generic cultural knowledge. ${ }^{3}$ Three systematic reviews published on the topic have reported positive effects of this training, including improved patient satisfaction, mutual understanding and adherence to treatment. ${ }^{5-7}$ Some authors recently criticised cultural competence, however, based on its superficial and often utilitarian understanding of the patient culture. ${ }^{8}$ Others advocate for more comprehensive approaches to cultural diversity in healthcare, such as cultural safety. ${ }^{9}$ 
Cultural safety is 'a space that is spiritually, socially, emotionally and physically safe for people; where there is no assault, challenge or denial of their identity, of who they are, and what they need'. ${ }^{10}$ This is an opportunity to overcome some of the limitations of cultural competence. ${ }^{11}$ Adding considerable value to other cultural approaches in healthcare, we found that cultural safety requires active participation of patients of non-dominant cultures in cocreating interventions that address their needs. ${ }^{12}$ Recent evidence suggests this type of training is linked to improved relationships between patients in non-dominant cultures and their health professionals, and to improved health outcomes. ${ }^{13}$

Culturally diverse Colombia is an ideal setting for cultural safety education, with potential lessons for other countries. Some $14 \%$ of the population belongs to minority groups (mostly black and Indigenous), ${ }^{14}$ and some $40 \%$ of the population seeks care in traditional and cultural health practices. ${ }^{15}$ Yet modern health services and their training programmes rely entirely on Western concepts of health and healing. As part of an effort to bridge the cultural divide between Western health services and the cultural preferences of the Colombian society, a recent initiative to introduce cultural safety in medical training engaged traditional medicine users, medical students and cultural safety experts to develop a consensus of key elements for a codesigned curriculum for cultural safety training. ${ }^{16}$ A challenge facing cultural safety education, however, is that health professionals are seldom motivated to interact with traditional health practices ${ }^{17}$ or to receive intercultural education, ${ }^{18}$ thus requiring innovative teaching strategies to overcome these challenges.

Game jams are participatory events that allow attendants to cocreate digital or board games in a timerestricted environment. ${ }^{19}$ These events have been used for other purposes beyond game design. Examples include the Health Games Challenge Game Jam in 2010; the Fukushima Game Jam, aimed at providing assistance after the 2011 earthquake and nuclear disaster ${ }^{20}$; and even the use of the game jam model as a research codesign method. ${ }^{21}$ We believe that game jams could provide a more engaging cultural safety learning experience for health professionals. Recent studies of game jam learning suggest likely effectiveness and engagement potential in university-level education. ${ }^{22}$ Laiti and collaborators ${ }^{23}$ suggested that game jams could promote self-discovery, reflections on identity and support the cultural identity of the Sami people from Finland.

We could find no published medical education research exploring the role of game jam learning. In preparation for a full-scale randomised controlled trial (RCT) to determine whether game jam learning is more effective than a standard lesson on cultural safety in terms of change in medical students' self-reported cultural safety intended behaviour, ${ }^{24}$ this pilot study attempted to explore (1) the acceptability and feasibility of conducting an RCT to assess game jam learning for cultural safety training of medical students, (2) the validity and reliability of our research instrument, and (3) pilot research methods and procedures.

\section{METHODS}

\section{Study design}

A parallel-group, two-arm, pilot RCT with a 1:1 allocation ratio compared participation in a game jam on cultural safety with a standard lesson on cultural safety, with student self-reported cultural safety intended behaviour as the outcome. We followed the CONSORT extension for randomised pilot and feasibility trials to report our results $^{25}$ (see online supplemental file 1 ). The protocol of the full-scale study is available elsewhere. ${ }^{26}$ The fullscale study is registered on ISRCTN registry (http://www. isrctn.com/ISRCTN14261595).

\section{Setting and participants}

The Faculty of Medicine at La Sabana University in Chia is located $24 \mathrm{~km}$ from Bogota, the capital of Colombia. La Sabana University is a private institution with 9000 undergraduate students. ${ }^{27}$ In 2017 , we invited a convenience sample of 79 final-year medical students taking a compulsory community health course, ${ }^{28}$ expecting to allocate at least 30 students to each arm. Our sample size was restricted by the availability of students due to other commitments. Inclusion criteria were (1) being a medical student taking the community health course and (2) giving informed consent. The single exclusion criterion was not wanting to participate in the study. Since $40 \%$ of Colombians seek care in cultural health practices, ${ }^{15}$ we anticipated that some students would be more open to the cultural safety approach than others. To allocate the participants to the study arms, we used stratified randomisation based on baseline cultural safety scores (lower and higher levels).

\section{Procedure}

The intervention was a game jam, ${ }^{19}$ a 1-day collaborative activity to codesign low-tech prototypes of educational games about cultural safety. The game jam comprised three parts: (1) a 1-hour preliminary lecture on cultural safety, (2) a 30 min lecture about game-design principles and (3) a 3.5-hour practical session to create educational games about cultural safety. We used the preliminary version of a medical curriculum we had developed in parallel to this pilot study to inform the lecture on cultural safety. ${ }^{16}$ The students worked in groups of four or five to create game prototypes, including narratives, objectives, game dynamics, rewards and penalties. We asked the students to bring game elements from their favourite board games and provided game materials such as game boards, playing cards, game money, dice, markers, pencils, glue and tokens. A video of the activity is available. ${ }^{29}$

The control group received a 1-hour PowerPoint-based conventional lesson on cultural safety in healthcare. This group also had 4 hours to study selected readings on cultural safety. We provided laptops with the readings 
for those students who did not have access to the class materials. The class for the control group included the same cultural safety concepts covered in the intervention group activities.

\section{Measures}

Acceptability

We explored the acceptability of the study activities for the medical students and for the academic staff. We used a standard definition of "whether the research is likely to be acceptable to potential participants ${ }^{30}$ to inform our understanding of acceptability. We assessed student interest and willingness to participate and to complete study activities, and we evaluated this with participant recruitment, retention and survey response rates. Our instrument also included an open-ended question at the end of the questionnaire to assess student perception of the activity. We analysed these responses using an inductive thematic analysis. ${ }^{31}$ We also aimed to determine whether the academic staff at La Sabana University would accept cultural safety training, given the reported reluctance of health professionals to support teaching of traditional and cultural health practices. ${ }^{17}$

\section{Feasibility}

We used Eldridge et $a l \mathrm{~s}^{32}$ definition of feasibility to ask: 'whether something can be done, should we proceed with it, and if so, how'. In the pilot RCT context, we explored the methodological and logistical factors that would make the full-scale study possible or not. For example, we explored whether using self-administered online questionnaires was feasible.

\section{Instrument}

Given that cultural safety is context specific, we developed a survey instrument based on the characteristics of our cultural context in Colombia. The research team developed a preliminary version of the instrument that a group of five cultural safety experts (four from Colombia and one from Canada) later reviewed and refined.

An online self-administered multiple-choice Likert-type questionnaire assessed the efficacy of the two teaching methods. A modified theory of planned behaviour ${ }^{33}$ provided a framework for our primary outcome. Seven statements explored the results chain (conscious knowledge, attitudes, positive deviation from negative subjective norms, change intention, sense of agency, discussion and change in practice/action (CASCADA) ) in response to real-life clinical cases depicting intercultural tensions. For example, the statement 'You know what the cultural safety approach is' indicated conscious knowledge. Each question had five response options ranging from 'strongly agree $=5$ ' to 'strongly disagree $=1$ '. The questions used to assess each component of the CASCADA model are available (online supplemental file 2). We added the scores of each of the seven questions to determine a cultural safety score. The students completed a baseline questionnaire, a second questionnaire immediately following the teaching session and a third questionnaire 4 months after the intervention.

\section{Validity of the instrument}

Jeffreys ${ }^{34}$ described an approach to improve the validity and reliability of the Transcultural Self-Efficacy Tool, an instrument to assess cultural competence education of health professionals. We followed this approach to increase the validity and reliability of our instrument. We followed a two-phase procedure to increase the content validity. ${ }^{35}$ First, an open question at the end of the questionnaire (how can we improve this instrument?) explored student opinions of the instrument. An inductive thematic analysis $^{31}$ of responses generated suggestions to adjust our survey. Second, we shared the adjusted version of the instrument by email with two general medical practitioners, one medical intern, six medical students and four cultural safety experts, all from Colombia. We asked the question: does our instrument assess cultural safety training? We adjusted the instrument according to their comments and reached a consensus on the content validity of the instrument.

To increase the construct validity of our instrument, we used the contrasted group approach, which explores the difference between two separate groups. ${ }^{34}$ Our stratified randomisation facilitated this. We tested the assumption that the greatest change in cultural safety score will be detected in individuals with a low score initially who have then been exposed to formalised cultural safety training. To increase the predictive validity of our instrument, we looked at the score difference between two timepoints. ${ }^{36}$ We compared the first timepoint with the last timepoint. We assumed that cultural safety is a dynamic construct that changes over time, and it is influenced by formalised exposure to cultural safety training.

\section{Reliability of the instrument}

Reliability explores the degree of accuracy and consistency in measurement. We explored the reliability of our instrument using the test-retest method, ${ }^{34}$ the most common approach to measure the stability of an assessment over time. ${ }^{37}$ Our assumption was that the students' scores would be stable across the second and third timepoints.

\section{Analysis}

A simple and paired t-test explored between-group and within-group differences, respectively. For the secondary analysis, we used probabilistic transitive closure of each CASCADA result chain. ${ }^{38}$ This allowed us to explore walks and blocks between the seven intermediate outcomes of the CASCADA model and to determine the cumulative net influence. In this way, we evaluated the CASCADA construct as a network of inter-related elements rather than an isolated outcome.

We used R Studio to convert each intermediate Likerttype outcome of the CASCADA results chain into binomial variables. We compared students who reported the greater effect against the remaining students (cut-off 
point (1 to 4$)$ vs (5)) with 1 as strongly disagree and 5 as strongly agree. We used the TABMAT command on CIETmap 2.0 V.1 $17^{39}$-an open-source software that uses the statistical programming language $\mathrm{R}$ - to generate a matrix of crude ORs between each pair of intermediate outcomes in the CASCADA model. Subsequently, we calculated the probabilistic transitive closure and uploaded the resulting adjacency matrix to yED V.3.19.1 to create cognitive maps representing the walks and blocks between the CASCADA elements.

To identify potential patterns of missing data that could hinder retention in the full-scale RCT, we stratified missing data by participant age, socioeconomic level, sex, place of birth and presence of family in rural areas for each timepoint. Finally, we conducted an inductive thematic analysis ${ }^{31}$ of the open-ended questions at the end of the questionnaire to identify themes describing (1) the students' perception of the activity (acceptability) and (2) the content validity of our instrument.

\section{Patient and public involvement}

The study received support from an advisory group composed of cultural safety experts, medical students and general physicians. The group helped to develop the research instrument, and we invited them to codesign the dissemination plan, funded by the Quebec Population Health Research Network.

\section{RESULTS}

A total of 79 students completed the baseline questionnaire, and we randomised these centrally to two arms. Only 64 students completed the second timepoint and 35 completed the third. Table 1 shows the sociodemographic characteristics of the participants, and figure 1 shows the schedule of enrolment, interventions and assessments of the pilot RCT.

Students randomised to the intervention arm reported a slightly higher cultural safety score after the intervention, not significant at the $5 \%$ level. Both intervention and control arms reported a higher cultural safety score after participating in the training sessions, with statistically significant differences comparing the pre and post timepoints. Tables 2 and 3 show the difference in cultural safety score between and within intervention groups by timepoint.

The net probabilistic transitive closure showed a good progression of the CASCADA intermediate outcomes for both the intervention and control groups after the intervention (figure 2). The intervention group showed a higher cumulative net influence (5.99) than the control group (5.86), and all arcs where positive.

\section{Perception of the activity}

Students in the intervention arm described the game jam experience in favourable terms. They highlighted the importance of a topic that is not offered in standard
Table 1 Sociodemographic characteristics of the participants of the study

\begin{tabular}{|c|c|c|}
\hline & $\begin{array}{l}\text { Standard } \\
\text { lesson } \\
(n=33)\end{array}$ & $\begin{array}{l}\text { Game jam } \\
(n=31)\end{array}$ \\
\hline & n (\%) & n (\%) \\
\hline \multicolumn{3}{|l|}{ Sex } \\
\hline Female & $20(61)$ & $18(58)$ \\
\hline Male & 13 (39) & $13(42)$ \\
\hline \multicolumn{3}{|l|}{ Place of birth } \\
\hline Bogotá & $18(54.6)$ & $20(64.5)$ \\
\hline Colombia, another city & $13(39.4)$ & $9(29)$ \\
\hline Venezuela & $2(6)$ & $2(6.5)$ \\
\hline \multicolumn{3}{|l|}{ Family in rural settings } \\
\hline Yes & $12(36.6)$ & $15(48.4)$ \\
\hline No & $21(63.4)$ & $14(45.2)$ \\
\hline Do not know & 0 & $2(6.4)$ \\
\hline \multicolumn{3}{|l|}{ Socioeconomic level } \\
\hline One (lowest) & 0 & 0 \\
\hline Two & $2(6)$ & 0 \\
\hline Three & $4(12.1)$ & $5(16.1)$ \\
\hline Four & $22(66.8)$ & $9(29)$ \\
\hline Five & $4(12.1)$ & $13(42)$ \\
\hline Six (highest) & $1(3)$ & $4(12.9)$ \\
\hline \multicolumn{3}{|c|}{ Family uses traditional medicine } \\
\hline Yes & $26(78.8)$ & $23(74.2)$ \\
\hline No & $7(21.2)$ & $8(25.8)$ \\
\hline Do not know & 0 & 0 \\
\hline \multicolumn{3}{|c|}{ Medicinal plants planted at home } \\
\hline Yes & 9 (27.3) & $13(42)$ \\
\hline No & $24(72.7)$ & $18(58)$ \\
\hline Do not know & 0 & 0 \\
\hline \multicolumn{3}{|c|}{ Student self-identifies as minority } \\
\hline Rural population/peasant & $1(3)$ & $3(9.6)$ \\
\hline No & 32 (97) & $28(90.4)$ \\
\hline Do not know & 0 & 0 \\
\hline \multicolumn{3}{|l|}{ Age (years) } \\
\hline Min & 21 & 21 \\
\hline Max & 30 & 30 \\
\hline Mean (SD) & $22.7 \pm 1.6$ & $23.3 \pm 1.8$ \\
\hline
\end{tabular}

medical training, and described game jam learning as an innovative strategy to learn through hands-on experience:

It was great learning about such an important and neglected topic [cultural safety], and it was even better learning through innovative approaches such as the game jam. (Student 11, female) 


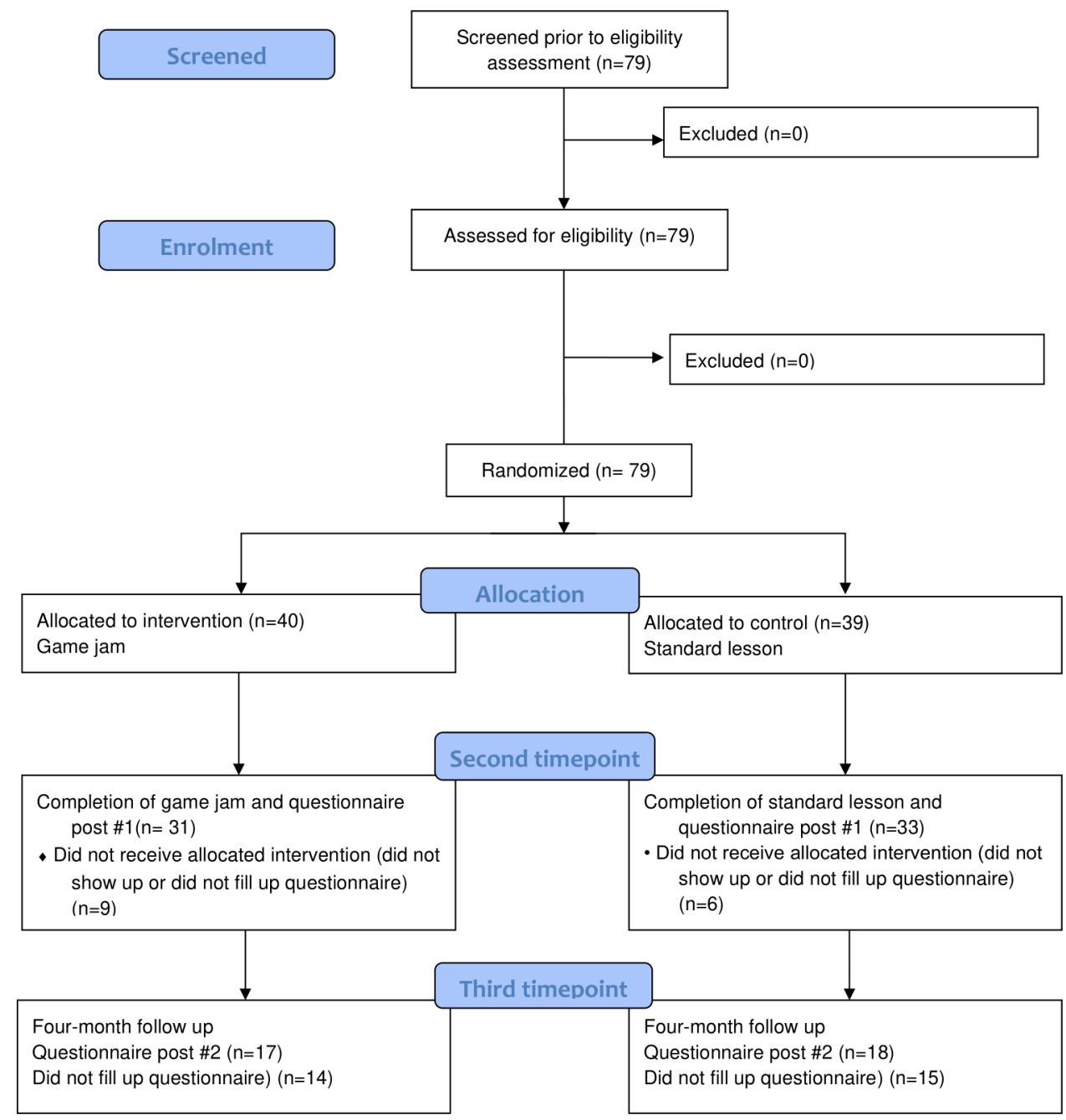

Figure 1 Schematic diagram of the pilot randomised controlled trial.

The experiential learning approach is the best way to learn. I learned a lot: cultural safety, game jam, innovation, and creativity! (Student 14, female)

We learned through creating the games because we used the knowledge that we acquired in the lecture. In this self-learning activity, we could apply what we learned through hands-on experience and playing. (Student 3, male)

Additionally, some medical students considered cultural safety training relevant to the multicultural context of Colombia and commented about its potential benefits:
It is worth having this training to educate integral physicians who are adapted to the cultural characteristics of the communities in Colombia. (Student 5 , male)

The lecture was fascinating, and I think that [cultural safety training] invites us to become more curious about our own culture, to be more inclusive towards our patients, and to accept cultural differences in our professional practice. (Student 18, female)

I think it is a topic that all medical students should learn because our country is extremely rich in terms of cultural diversity. It is very common to see patients with different cultural healing practices. (Student 28, female)

\begin{tabular}{|c|c|c|c|c|c|}
\hline & Standard lesson & Game jam & Difference & $95 \% \mathbf{C l}$ & $\mathbf{n}$ \\
\hline Preintervention & 23.3 & 23 & 0.3 & -1.4 to 2 & 64 \\
\hline Postintervention 2 & 26.8 & 26.5 & 0.3 & -2.4 to 3 & 35 \\
\hline
\end{tabular}


Table 3 Difference of cultural safety score within intervention groups

\begin{tabular}{|c|c|c|c|c|c|}
\hline & Preintervention & Postintervention 1 & Difference* & $95 \% \mathrm{Cl}$ & $\mathbf{n}$ \\
\hline Total & 23.1 & 26.3 & -3.2 & -4.3 to -2 & 64 \\
\hline Standard lesson & 23.3 & 25.9 & -2.6 & -4. To -1.1 & 33 \\
\hline \multirow[t]{2}{*}{ Game jam } & 23 & 26.9 & -3.9 & -5.6 to -2.1 & 31 \\
\hline & Preintervention & Postintervention 2 & Difference & $95 \% \mathrm{Cl}$ & $\mathbf{n}$ \\
\hline Total & 23.1 & 26.7 & -3.6 & -4.7 to -1.6 & 35 \\
\hline Standard lesson & 23.9 & 26.8 & -2.9 & -5.3 to -0.4 & 18 \\
\hline \multirow[t]{2}{*}{ Game jam } & 23 & 26.6 & -3.6 & -5.4 to -0.9 & 17 \\
\hline & Postintervention 1 & Postintervention 2 & Difference & $95 \% \mathrm{Cl}$ & $\mathbf{n}$ \\
\hline Total & 26.3 & 26.7 & -0.4 & -1.7 to 1 & 35 \\
\hline Standard lesson & 25.7 & 26.8 & -1.1 & -3.1 to 0.9 & 18 \\
\hline Game jam & 26.9 & 26.6 & 0.3 & -1.6 to 2.4 & 17 \\
\hline
\end{tabular}

*Significant differences are shown in bold font.

The students suggested an increase in the game building period since the 3.5-hour block was not enough to fully create and test an educational game. Similarly, the students recommended having facilitators to ensure better progress of the game jam.

\section{Content validity of the instrument}

The medical students, general practitioners and medical interns recommended changes in the instrument for the full-scale study. They suggested better wording of questions and shorter statements (some questions had two statements), as well as adding the definition of some traditional concepts in the questionnaire, for example, 'atole'-traditional hot corn-based beverage of Mesoamerican origin. ${ }^{40}$ The cultural safety experts suggested using the term 'yerbas medicinales' (medicinal herbs) instead of 'plantas medicinales' (medicinal plants) throughout the questionnaire. According to these experts, 'yerbas medicinales' may better reflect the traditional use of medicinal plants.

Intervention

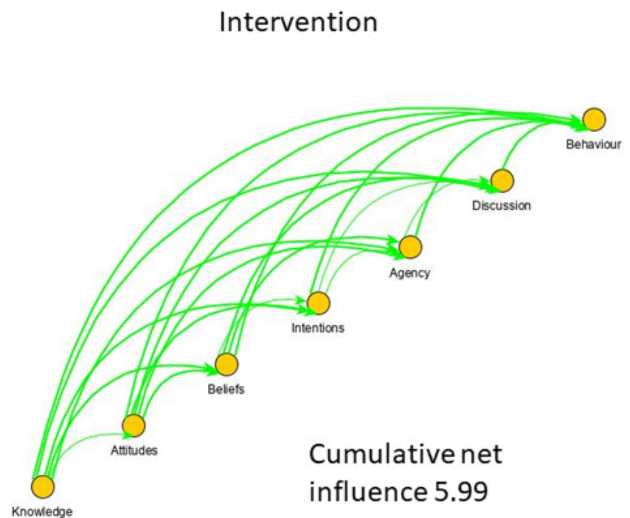

\section{Construct and predictive validity}

For both the second and third timepoints, the greatest change in cultural safety score was among students with low cultural safety score initially who were then exposed to cultural safety training (figure 3). This confirmed the contrasted group assumption. Since we observed a significant increase in the cultural safety score comparing the baseline and the last timepoint, our findings support the assumption that the cultural safety score is a dynamic construct that changes over time, and it is influenced by exposure to cultural safety training (figure 3 ).

\section{Reliability of the instrument}

The difference between the second and the third timepoints revealed the stability of the instrument (figure 3). We did not detect a significant difference in student scores between the second and third timepoints, which corroborated the test-retest reliability assumption (table 3). Finally, we did not identify patterns of missing

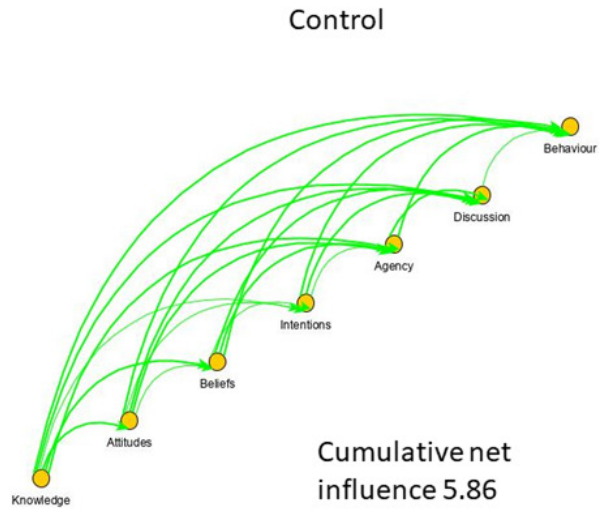

Figure 2 Net probabilistic transitive closure of the CASCADA results chain of medical students' responses in control and intervention groups in the pilot randomised controlled trial. CASCADA, conscious knowledge, attitudes, positive deviation from negative subjective norms, change intention, sense of agency, discussion and change in practice/action. 


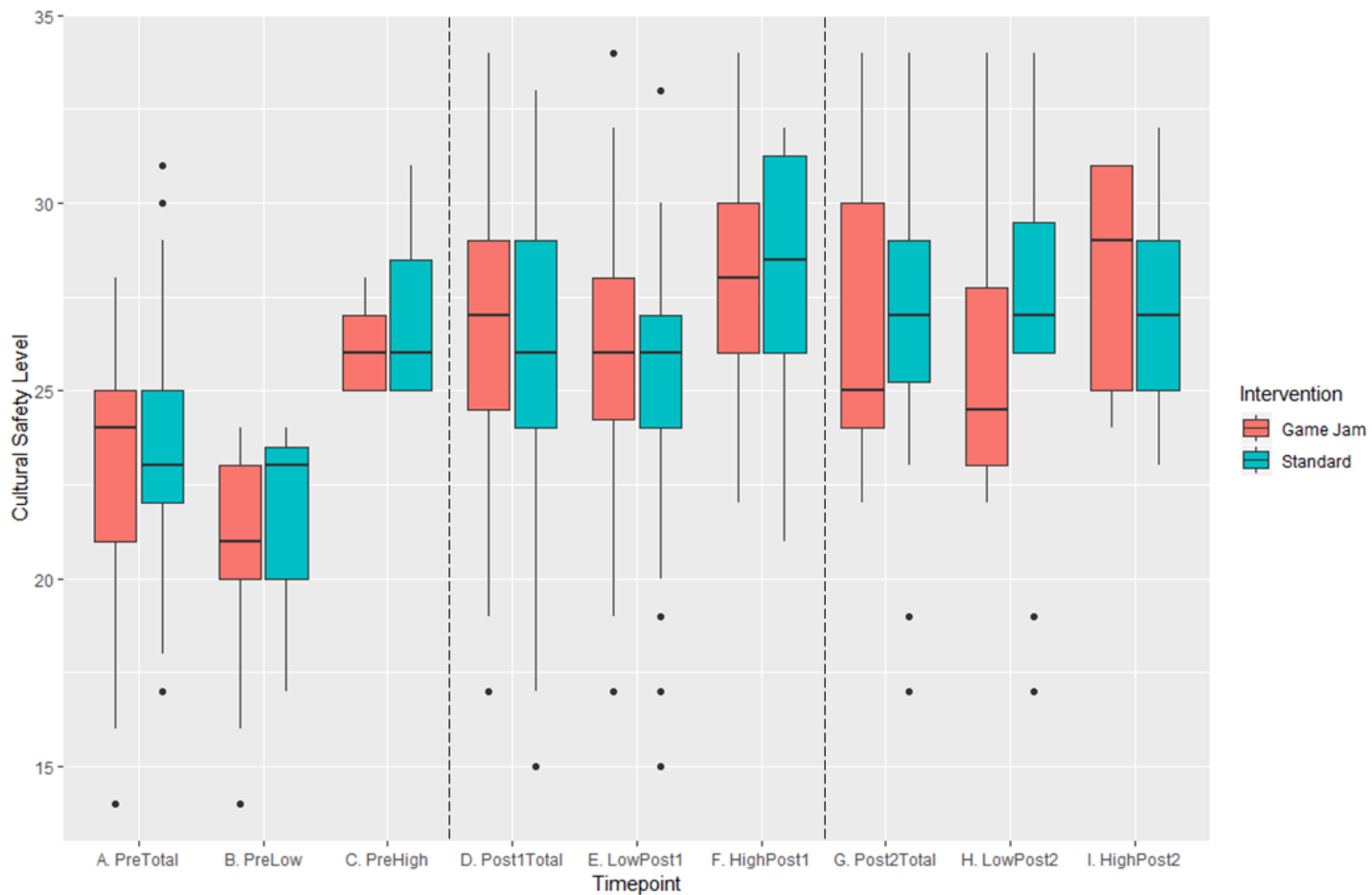

Figure 3 Differences in cultural safety score between intervention groups by timepoints. The figure compares the scores of cultural safety between the study intervention and control groups. For each timepoint, we show the total score, the score for students with a low score preintervention and the score for students with a high score preintervention. Low preintervention scores are those below the median, and high preintervention scores are those above the median. The horizontal lines in the figure are the median values for the group at that timepoint.

data that could hinder retention in the full-scale RCT along timepoints.

\section{DISCUSSION}

Compared with the control group, students allocated to the intervention arm reported a slightly higher cultural safety score after the intervention, although this difference was not statistically significant. Pilot RCT studies, however, are not appropriate to support statistical inferences and should be rather focused on acceptability and feasibility of interventions and data collection/analysis methods. ${ }^{41}$ Our pilot study supported the acceptability and feasibility of cultural safety training through game jam learning. It allowed us to gain skills required to conduct a full-scale game jam and RCT, to test research methods and procedures, and to explore the validity and reliability of our instrument. It was also a valuable opportunity to garner the support of the faculty of the university for the full-scale study.

\section{Acceptability and feasibility of cultural safety training and game jam learning}

Participating medical students reported that cultural safety education is relevant to the multicultural context of Colombia, and they commented about the potential benefits of this training. An earlier small-scale communitybased learning intervention ${ }^{42}$ helped Colombian medical students become aware of the relevance of this type of training in a multicultural setting such as Colombia. In another community-based learning experience, ${ }^{43}$ Colombian medical students reported four areas of change after receiving cultural safety training: increased respect for traditional medicine, recognition of traditional medicine as part of their cultural heritage and identity, a growing interest in traditional medicine and openness to incorporate traditional medicine in healthcare. Our full-scale trial $^{26}$ will help to explore the transformative potential of cultural safety training. While medical students reported that the cultural safety training was important, attrition of students throughout the pilot indicated the need for recruiting facilitators to support retention in the full-scale study.

Moore et al argued that pilot studies are necessary to establish feasibility. ${ }^{44}$ The idea of game jams to improve learning is recent ${ }^{45}$ and, to the best of our knowledge, this is the first experience using a game jam to train medical students. In the open-ended questions, the medical students described the game jam as an innovative strategy to learn through hands-on experience. Some authors recently reported that game jam participation could improve performance of computing students, ${ }^{19} 22$ promoting learning through self-discovery and reflections on identity. ${ }^{23}$ The full-scale RCT will shed further light on the learning potential of game jams in medical education.

Pilot studies can also reveal local attitudes or interests that might affect the research process. ${ }^{46}$ Mudur $^{17}$ described opposition from Western trained physicians when the Indian Ministry of Health proposed introducing 
courses on traditional medicine for medical students. Prior to study initiation, we presented the project to the Academic Commission, the Sub-committee for Research and the Department of Epidemiology in three separate meetings at the Faculty of Medicine at La Sabana University. The academic staff requested us to explore the validity and reliability of the instrument before the full-scale study and to ensure that the intervention and control arms were exposed to the same cultural safety concepts. The meetings gained the support of the faculty at the university, which authorised the conduct of the fullscale RCT.

\section{Research methods and procedures}

Game jams are complex and chaotic, often lacking identifiable structure or form, and this makes them unpredictable. ${ }^{47}$ Experts highlight the role of hands-on practice to master game jam facilitation skills. Macklin $e t a l,{ }^{48}$ for example, mentioned that 'game jams are best mastered through practice.[...] With each one, you'll add ideas, style, and specifics that will make the next one you do even better' (p209). Our pilot RCT allowed us to develop the skills to conduct a successful full-scale RCT with a game jam intervention.

Van Teijlingen $e t a t^{41}$ found a pilot study identified potential problems in a research plan on maternity care. It showed the proposed procedure of distributing questionnaires would not work for a full-scale study. ${ }^{41}$ Our pilot helped us to test mobile devices for the self-administered questionnaires. Since all students had access to a personal device (smartphone, tablet or computer), we confirmed this method would be feasible and convenient in the fullscale study. We also determined the time, materials and staff we would require. Students requested, for example, the game jam last longer than 5 hours.

Pilot studies also allow researchers, including those in training, to gain experience with research methods, procedures and data analysis techniques. ${ }^{414}$ We practised stratified randomisation by level of training on cultural safety at baseline. Similarly, we explored data analysis using probabilistic transitive closure. Andersson and collaborators used fuzzy transitive closure to explore gender-specific CASCADA intermediate outcomes underlying dengue prevention in the Camino Verde trial. ${ }^{38}$ They illustrated gender differences in the overall influence of the intervention potentially due to higher participation and engagement of women. In transitive closure, every relationship in the map contributes to the summary weight of a walk through a multiplicative function. The approach is appropriate when the number of factors of the model is preset-for example, in the fixed-length CASCADA results chain-and when the weights of relationships between concepts can be interpreted as probabilities. ${ }^{49}$ To our knowledge, the present study is only the second application of transitive closure to examine group-specific intermediate outcomes in behaviour change interventions and the first to do so with probabilistic transitive closure.

\section{Validity and reliability of our research instrument}

Our recent scoping review could identify no validated instruments to assess cultural safety interventions. ${ }^{50}$ Since cultural safety responds to the specific cultural needs of each cultural setting, ${ }^{12}$ research instruments should also reflect the specific characteristics of each context. In piloting a preliminary version of our instrument, we used stakeholder opinions on how to improve the questions of the survey to enhance the content validity of our instrument. We explored the construct and predictive validity, as well as the reliability of our instrument in preparation for the full-scale study.

\section{Limitations}

A pilot focused entirely on the validity and reliability of the instrument might have achieved more in those domains. The objectives of our study, however, went beyond exploring the performance of our instrument, and we had to balance our time and resources to cover the complex elements and skills to conduct the innovative full-scale study. Additional methods to increase the validity of our instrument, for example, factor analysis, ${ }^{51}$ could be explored in the future.

We did not include a qualitative evaluation of the impact of the intervention in the clinical practice of the medical students as will the full-scale study. We propose to examine the most significant change ${ }^{52}$ in the clinical practice of the medical students and interns after participating in cultural safety training. This will help us to identify unintended effects of the intervention and help to understand the experience of the intervention in healthcare education.

Our pilot study detected considerable interaction between students, with resulting contamination between the intervention and control groups. This could affect estimation of impact in our future full-scale RCT. We adjusted the protocol of the full-scale study to include facilitators, who will help to minimise communication and subsequent contamination between the two study arms. The facilitators will also help to address participant attrition in the full-scale study.

Students described cultural safety training and the game jam experience in favourable terms. A well-recognised limitation in medical education research, however, is its risk of social desirability bias. ${ }^{53}$ Medical students could respond with the most socially desirable answers rather than their own point of view. We encouraged students to be sincere when writing down their opinions and assured them that their answers would not have any impact on their performance. We recognise, nonetheless, social desirability bias is impossible to eliminate completely.

\section{CONCLUSION}

Cultural safety is a relevant approach to medical education in Colombia, where training of physicians is disconnected from the cultural characteristics of the country. Our pilot study suggested cultural safety training is 
feasible and acceptable to Colombian medical students when delivered through game jam learning. We gained the skills required to conduct a full-scale study, piloted research methods and data analysis techniques, improved both our instrument and intervention, identified logistical problems that may hinder our research endeavour and garnered the support of the faculty of the university for the full-scale study. Researchers and educators may find our results informative in the design of RCTs assessing educational interventions. We are using the lessons learnt in our study to conduct an ongoing full-scale study with 400 medical students in Colombia. ${ }^{24} 26$

\section{Twitter Juan Pimentel @juanppimentel}

Acknowledgements Drs Germán Zuluaga, Andrés Isaza, Andrés Cañón, Carolina Amaya, Iván Sarmiento, Claudia Jaimes and Camila Kairuz provided advice to improve the content validity of our instrument. Drs Álvaro Romero, Yahira Guzmán, Camilo Correal, Francisco Lamus, Erwin Hernández and Rosa Durán supported the study activities at La Sabana University. Cassandra Laurie helped proofread the final version of the manuscript and supported its write-up.

Contributors This study is part of the PhD work of JPP. NA is the supervisor and $A C$ is the cosupervisor of JPP. NA and AC advised on the development of the study. JPP led the study design, coordinated the fieldwork and drafted this paper. All authors made adjustments, and read and approved the final manuscript.

Funding This study was financed by two travel awards awarded to the first author by McGill University: the Norman Bethune Award for Global Health and the Graduate Mobility Award. The first author is supported by the CeiBA Foundation (Colombia) and the Fonds de recherche du Québec-Santé (Canada). This did not influence the design, execution or publication of the study.

Competing interests None declared.

Patient consent for publication Not required.

Ethics approval The institutional review board of the McGill's Faculty of Medicine (approval number A05-B37-17B) and the subcommittee for research of the Faculty of Medicine at La Sabana University (approval number 445) provided ethical clearance for this pilot study. All participants signed a written informed consent before proceeding with any research activity.

Provenance and peer review Not commissioned; externally peer reviewed.

Data availability statement The data sets generated and/or analysed during the current study are available from the corresponding author on reasonable request.

Supplemental material This content has been supplied by the author(s). It has not been vetted by BMJ Publishing Group Limited (BMJ) and may not have been peer-reviewed. Any opinions or recommendations discussed are solely those of the author(s) and are not endorsed by BMJ. BMJ disclaims all liability and responsibility arising from any reliance placed on the content. Where the content includes any translated material, BMJ does not warrant the accuracy and reliability of the translations (including but not limited to local regulations, clinical guidelines, terminology, drug names and drug dosages), and is not responsible for any error and/or omissions arising from translation and adaptation or otherwise.

Open access This is an open access article distributed in accordance with the Creative Commons Attribution Non Commercial (CC BY-NC 4.0) license, which permits others to distribute, remix, adapt, build upon this work non-commercially, and license their derivative works on different terms, provided the original work is properly cited, appropriate credit is given, any changes made indicated, and the use is non-commercial. See: http://creativecommons.org/licenses/by-nc/4.0/.

\section{ORCID iD}

Juan Pimentel http://orcid.org/0000-0002-6842-3064

\section{REFERENCES}

1 Allan B, Smylie J. First peoples, second class treatment: the role of racism in the health and well-being of Indigenous peoples in Canada. Toronto, ON: Wellesley Institute, 2015.
2 Liaison Committee on Medical Education. Functions and structure of a medical school: standards for accreditation of medical education programs leading to the MD degree. Washington, DC: Liaison Committee on Medical Education, 2017.

3 Kirmayer LJ. Rethinking cultural competence. Transcult Psychiatry 2012;49:149-64.

4 Cross TL, Bazron BJ, Dennis KW. Towards a culturally competent system of care: a monograph on effective services for minority children who are severely emotionally disturbed. Washington, DC 1989.

5 Horvat L, Horey D, Romios P. Cultural competence education for health professionals. Cochrane Database Syst Rev 2014;CD009405:1-100.

6 Lie DA, Lee-Rey E, Gomez A, et al. Does cultural competency training of health professionals improve patient outcomes? A systematic review and proposed algorithm for future research. $J$ Gen Intern Med 2011;26:317-25.

7 Beach MC, Price EG, Gary TL, et al. Cultural competence: a systematic review of health care provider educational interventions. Med Care 2005;43:356-73.

8 Pon G. Cultural competency as new racism: an ontology of forgetting. J Progress Hum Serv 2009;20:59-71.

9 Brascoupe S, Waters C. Cultural safety: exploring the applicability of the concept of cultural safety to Aboriginal health and community wellness. J Aborig Heal 2009;5:6-41.

10 Williams R. Cultural safety--what does it mean for our work practice? Aust N Z J Public Health 1999;23:213-4.

11 Curtis $\mathrm{E}$, Jones $\mathrm{R}$, Tipene-Leach $\mathrm{D}$, et al. Why cultural safety rather than cultural competency is required to achieve health equity: a literature review and recommended definition. Int $J$ Equity Health 2019;18:174.

12 Blanchet Garneau A, Pepin J. La sécurité culturelle : une analyse du concept. Rech Soins Infirm 2012;4:22.

13 Kurtz DLM, Janke R, Vinek J, et al. Health sciences cultural safety education in Australia, Canada, New Zealand, and the United States: a literature review. Int J Med Educ 2018;9:271-85.

14 Intell. Agency. The world Factbook - Colombia. cent, 2018. Available: https://www.cia.gov/library/publications/the-worldfactbook/geos/co.html

15 World Health Organization. WHO traditional medicine strategy 2002-2005. 2002:1-74., 2002. Available: https://apps.who.int/ medicinedocs/en/d/Js2297e/

16 Pimentel J, Zuluaga G, Isaza A. Curriculum co-design for cultural safety training of medical students in Colombia: protocol for a qualitative study. In: Costa AP, Reis LP, Moreira A, eds. Computer supported qualitative research. Cham, Switzerland: Springer, 2019: 102-9.

17 Mudur G. Indian doctors decry proposal to teach traditional medicine. BMJ 2001;323:1090.

18 Dykes DC, White AA. Culturally competent care pedagogy: what works? Clin Orthop Relat Res 2011;469:1813-6.

19 a PJ, Chastine J, O'Donnell C. Game jams. Int J Game-Based Learn 2012;2:51-70.

20 Shin K, Kaneko K, Matsui Y. Localizing global game jam: designing game development for collaborative learning in the social context. in: lecture notes in computer science (including subseries lecture notes in artificial intelligence and lecture notes in bioinformatics. Berlin: Springer, 2012: 117-32.

21 Deen M, Cercos R, Chatman A. Game jam:[4 research]. In: CHI'14 extended abstracts on human factors in computing systems. Toronto, ON: ACM, 2014: 25-8.

22 Fowler A, Ni X, Preston J. The pedagogical potential of game jams. In: In: proceedings of the 19th annual SIG conference on information technology education - SIGITE '18. New York, USA: ACM Press, 2018: 112-6.

23 Laiti O, Harrer S, Uusiautti S, et al. Sustaining intangible heritage through video game storytelling - the case of the Sami Game Jam. Int $J$ of Stud 2021;27:296-311.

24 Pimentel J, Cockcroft A, Andersson N. Impact of game jam learning about cultural safety in Colombian medical education: a randomised controlled trial. BMC Medical Education 2019;21:1-12 https:// bmcmededuc.biomedcentral.com/articles/10.1186/s12909-02102545-7

25 Eldridge SM, Chan CL, Campbell MJ, et al. Consort 2010 statement: extension to randomised pilot and feasibility trials. Pilot Feasibility Stud 2016;2:64.

26 Pimentel J, Cockcroft A, Andersson N. Impact of Co-Designed game learning on cultural safety in Colombian medical education: protocol for a randomized controlled trial. JMIR Res Protoc 2020;9:e17297.

27 La Sabana University. The University in figures, 2018. Available: https://www.unisabana.edu.co/nosotros/la-sabana-en-cifras/ 
28 Lamus-Lemus F, Correal-Muñoz C, Hernandez-Rincon E, et al. The pursuit of healthier communities through a community health medical education program. Educ Health 2017;30:116.

29 Pimentel J, Pimentel M, Andersson N. Learning by jamming: codesign of serious games by medical students in Colombia - Pilot game jam., 2017. Available: https://www.youtube.com/watch?v= wb8xBskthMg\&feature=youtu.be

30 National Institute for Health Research, 2018. Available: http://www. netscc.ac.uk/getting_involved/PDFs/PPI_Glossary_of_research_ terms_v3.pdf [Accessed October 11, 2018].

31 Braun V, Clarke V. Using thematic analysis in psychology. Qual Res Psychol 2006;3:77-101.

32 Eldridge SM, Lancaster GA, Campbell MJ, et al. Defining feasibility and pilot studies in preparation for randomised controlled trials: development of a conceptual framework. PLoS One 2016;11:e0150205.

33 Andersson $\mathrm{N}$. Building the community voice into planning: 25 years of methods development in social audit. BMC Health Serv Res 2011;11 Suppl 2:S1.

34 Jeffreys MR. Preceded by: Jeffreys MR. Tools for assessment and evaluation. In: Teaching cultural competence in nursing and health care : inquiry, action, and innovation. New York: Springer Publishing Company, 2015: 89-194.

35 Waltz CF, Strickland O, Lenz ER. Measurement in nursing and health research. 3rd ed. New York, NY: Springer Pub, 2005.

36 Basalan I, Temel AB, Chen J. Transcultural self-efficacy Tool (TSET). In: Jeffreys M, ed. Teaching cultural competence in nursing and health care. Ann Arbor MICH, USA: ProQuest Ebook Central, 2015: 91-194.

37 Multon KD, Reliability T-R. Test-retest reliability. In: Salkind NJ, ed. Encyclopedia of research design. Thousand Oaks: SAGE Publications, Inc, 2010: 1496-8.

38 Andersson N, Beauchamp M, Nava-Aguilera E, et al. The women made it work: fuzzy transitive closure of the results chain in a dengue prevention trial in Mexico. BMC Public Health 2017;17:408.

39 Andersson N, Mitchell S. Epidemiological geomatics in evaluation of mine risk education in Afghanistan: introducing population weighted raster maps. Int J Health Geogr 2006;5:1.

40 Wikipedia. Atole, 2020. Available: https://en.wikipedia.org/wiki/Atole

41 Van Teijlingen ER, Rennie AM, Hundley V, et al. The importance of conducting and reporting pilot studies: the example of the Scottish births survey. J Adv Nurs 2001;34:289-95.
42 Pimentel J, Sarmiento I, Zuluaga G, et al. What motivates medical students to learn about traditional medicine? A qualitative study of cultural safety in Colombia. Int J Med Educ 2020;11:120-6.

43 Pimentel J, Kairuz C, Merchán C. The experience of Colombian medical students in a pilot cultural safety training program: a qualitative study using the most significant change technique. Teach Learn Med 2020:1-9.

44 Moore CG, Carter RE, Nietert PJ, et al. Recommendations for planning pilot studies in clinical and translational research. Clin Transi Sci 2011;4:332-7.

45 Fowler A, Khosmood F, Arya A. The global game jam for teaching and learning. In: proceedings of 4th annual conference of computing and information technology research and education New Zealand 2013:1-7.

46 van Teijlingen E, Hundley V. The importance of pilot studies. Nurs Stand 2002;16:33-6.

47 http://muep.mau.se/bitstream/handle/2043/17047/20140228 TorstenHansson_paper_EnhancingGameJamExperiences 1.52MB. pdf?sequence=2\&isAllowed=yMay 4, 2021 Hansson T. Enhancing game jam experiences: finding more productive and focused group work interactions through establishing a framework. Malmö, Sweden, Malmö University, 2014.

48 Macklin C, Martin J, Dikkers S. Planning your game jam: game design as a gateway drug. Mob Media Learn 2012:203-18.

49 Niesink P, Poulin K,ajnaŠajna M.Computing transitive closure of bipolar weighted digraphs. Discrete Applied Mathematics 2013;161:217-43.

50 Pimentel J, Arias A, Ramírez D, et al. Game-Based learning interventions to foster cross-cultural care training: a scoping review. Games Health J 2020;9:164-81.

51 Sellbom M, Tellegen A. Factor analysis in psychological assessment research: common pitfalls and recommendations. Psychol Assess 2019;31:1428-41.

52 Dart J, Davies R, A Dialogical DR. A Dialogical, Story-Based evaluation tool: the most significant change technique. American Journal of Evaluation 2003;24:137-55.

53 Gozu A, Beach MC, Price EG, et al. Self-Administered instruments to measure cultural competence of health professionals: a systematic review. Teach Learn Med 2007;19:180-90. 